\title{
Abstract 3064: Importance of circulating tumor cells in newly diagnosed colorectal cancer
}

\author{
Guus van Dalum, Marco R. de Groot, Gerrit-Jan Stam, Loes F.A. Scholten, Walter \\ J.B. Mastboom, Istvan Vermes, Arjan G.J. Tibbe and Leon W.M.M. Terstappen
}

DOI: 10.1158/1538-7445.AM2014-3064 Published October 2014

Proceedings: AACR Annual Meeting 2014; April 5-9, 2014; San Diego, CA

\section{Abstract}

Background: The presence of circulating tumor cells (CTC) is associated with poor prognosis in patients with metastatic colorectal cancer (CRC). This study was conducted to determine if the presence of CTC prior to surgery and during follow-up in patients with newly diagnosed nonmetastatic CRC identifies patients who are at risk for disease recurrence.

Methods: In a prospective single center study 183 patients with newly diagnosed nondisseminated CRC scheduled for surgery were enrolled from 2003 till 2008 and followed up for a median of 5.1 years. CTC were enumerated with the CellSearch System in 4 aliquots of $7.5 \mathrm{ml}$ of peripheral blood before and after surgery (1-26 weeks), after adjuvant therapy and 1, 2, 3 and 4 years after surgery.

Findings: $\geq 1 \mathrm{CTC} / 30 \mathrm{ml}$ of blood were detected in $44(24 \%)$ patients before surgery. CTC frequency did not change significantly at the time points after surgery. Patients with CTC before surgery had a significant decrease in Recurrence Free Survival (RFS, logrank test $p=0.014$ ) and Colon Cancer Related Survival (CCRS, $p=0.002$ ). Five year RFS dropped from $75 \%$ to $61 \%$ and five year CCRS from $83 \%$ to $69 \%$ for patients with CTC before surgery. In a multivariate analysis of CTC, T-Stage and N-stage, the presence of CTC and N-stage remained as significant factors for RFS and CCRS. Surprisingly the presence of CTC after surgery was not significantly associated with RFS and CCRS whereas CTC 2-3 years after surgery was again significantly associated with RFS and CCRS.

Interpretation: The presence of CTC in patients with stage I-III CRC before surgery is associated with a significant reduction RFS and CCRS. Although similar amounts of CTC were detected within 3 months after surgery they were not associated with RFS or CCRS. In contrast CTC were again highly significant for RFS and CCRS 2-3 years after surgery. These findings suggest a role of CTC detection, to assess which patients need adjuvant treatment. To implement CTC detection in the non-metastatic setting a validated CTC detection technology is needed with increased sensitivity and specificity.

Citation Format: Guus van Dalum, Marco R. de Groot, Gerrit-Jan Stam, Loes F.A. Scholten, Walter J.B. Mastboom, Istvan Vermes, Arjan G.J. Tibbe, Leon W.M.M. Terstappen. Importance of circulating tumor cells in newly diagnosed colorectal cancer. [abstract]. In: Proceedings of the 105th Annual Meeting of the American Association for Cancer Research; 2014 Apr 5-9; San Diego, CA. Philadelphia (PA): AACR; Cancer Res 2014;74(19 Suppl):Abstract nr 3064. doi:10.1158/1538-7445.AM2014-3064 